\title{
Measures to analyse sustainability in military workforce structures
}

\author{
C. Pike $^{\mathrm{a}}$ and W. Moran ${ }^{\mathrm{b}}$ \\ a Joint and Operations Analysis Division, Defence Science and Technology Group, Fishermans Bend, Victoria \\ ${ }^{\mathrm{b}}$ Department of Electrical \& Electronic Engineering, University of Melbourne, Parkville, Victoria \\ Email: Cameron.Pike@dst.defence.gov.au
}

\begin{abstract}
For defence services to provide effective capability, it is important that enough suitably qualified personnel are available at the right times. Essential to this, is a workforce structure that sustainably meets the personnel requirement. Specifically, an adequate number of positions in the different ranks is needed, as well as sufficient promotions and recruitment to sustain those positions over time, given the constraints on the workforce. In this paper, we mathematically explore the relationship between these parameters, describe how to determine whether a workforce structure is sustainable or not, and detail what changes may need to be made to achieve a healthy structure.

Here, sustainability means that the requirement profile - the number of positions in each rank at each time — can be achieved without excesses or shortfalls of personnel. We critique a simple linear programming (LP) optimisation formulation that was applied to Royal Australian Navy (RAN) test problems, and the unexpected results that it produced. In particular, it resulted in large oversupplies in lower ranks early in a testing period of 20 years. By using a cost function that penalised oversupply and undersupply with different weightings, we found that such oversupplies were necessary in order to avoid significant shortfalls of personnel in higher ranks and later years.
\end{abstract}

This provided the motivation for the main contribution of this paper: an exploration of why the oversupply was necessary and equations that could assist workforce planners to quantify the magnitude of this oversupply. This involved assessing how specific factors play a role in the sustainability of workforce structures. Backpropagation of required numbers of personnel through the ranks provided the necessary number of promotions at each rank and time in order to achieve that requirement. We refer to this number of promotions as the Required Production Number (RPN). An analysis of the distribution of time in rank permits calculation of how many promotion-eligible personnel could be produced at each rank and time. We refer to this number of producible personnel as the Suppliable Production Number (SPN).

When the RPN exceeds the SPN, one of two functionally equivalent situations has to happen to avoid future shortfalls in other ranks as predicted by the model: oversupplying a rank or increasing the requirement of a rank. We quantify the extent to which the requirement needs to be increased. Conversely, when the RPN is less than the SPN, the requirement can be met without excesses or shortfalls of personnel, but personnel will spend longer in a rank before promotion. Calculations to quantify the expected time to promotion are also described. This is an important consideration as personnel waiting too long for promotion may result in decreased morale and an increase in personnel leaving the workforce.

These simple and practical measures allow workforce planners to draw insights into their workforce structure. In particular, they can determine whether and why the requirement in particular ranks is inadequate to supply higher ranks and assess whether and why personnel are spending excessive amounts of time in ranks before promotion. This contrasts with LP optimisation results, which can show these effects, but not easily and directly explain how the workforce parameters influence them. We also see in this work that two different requirements emerge: the functional requirement: the number of personnel the defence service needs to operate, and the structural requirement, which is the workforce structure needed in order to ensure that the functional requirement can be sustainably met, and that defence capability is maintained.

Keywords: Workforce planning, workforce structure, military workforce, force structure, sustainability 


\section{INTRODUCTION}

The Royal Australian Navy (RAN) is undergoing significant workforce growth through its acquisition of new platforms. It is crucial that enough trained personnel be available to operate these platforms when introduced. It is also important that the workforce is structured so as to maintain this availability of sufficient personnel for these platforms over the long term (we examine periods of 20 years or longer). This issue of ensuring that the workforce is structured effectively for sustainability is an issue faced by all defence forces.

This work arose out of an effort to assist the RAN in determining optimal recruitment and promotion numbers so that the supply of personnel would be expected to meet the demand for positions in Navy's growing workforce. An optimiser was developed and applied on parts of the RAN workforce. It was found, however, that in some cases, the predicted supply for recruitment and promotions determined by the optimiser significantly exceeded the operational requirement. This was particularly true in lower ranks at early times in the analysis period, and occurred predominantly in situations of high workforce growth, high numbers of personnel leaving the workforce, or both. If the requirement was not exceeded in these cases, then shortfalls would occur in higher ranks at later times. The focus of this paper is an analysis of why certain workforce structures exhibit this behaviour and development of measures to quantify the extent to which this happens.

Many different workforce models have been developed by researchers; we mention just three: Gass (1991), Hall (2009), Scales et al. (2011). Each has different levels of fidelity and captures different aspects of interest. Because our aim is to analyse the relationship between a few key workforce parameters and supply, we apply a relatively simple and generic workforce model: the workforce consists simply of a progression of ranks with limited promotion criteria and a deterministic wastage rate of personnel leaving the workforce. The model can represent an entity as small as an individual trade, or as large as an entire defence service. It is designed with time frames of multiple decades in mind. We do not consider individual positions, units, and training, although the outcomes presented here are likely also relevant to models with these higher levels of fidelity.

The primary purpose of this work is not to produce optimal recruitment and promotion policies, though we do describe a basic Linear Programming (LP) formulation to obtain these policies so as to explore the relationship between supply, and workforce structure and its properties. Researchers have used several different techniques for this purpose, including mathematical/stochastic programming (Bastian et al., 2020), simulation-based system dynamics (Turan et al., 2019), mixed integer linear programming (Horn and Gomez-Iglesias, 2016), discrete event simulation (Moorhead et al., 2008), differential equations (Ozakawa et al., 2018), and Markov modelling (Filinkov et al., 2011).

Our motivation is to explain these optimisation results by mathematically analysing workforce structure for different parameters of requirement, wastage, and minimum time in rank. This is related to force structure analysis. Military workforce structures have been modelled by Wesolkowski and Eisler (2015), Filinkov et al. (2011), and Weigel and Wilcox (1993). Unlike force structure planning, we do not look at relating personnel requirement to capabilities and operations, but rather whether supplying the requirement of positions, as generated by force structure planning, is mathematically sustainable. This means analysing the pyramid-like rank structure of the workforce and identifying whether each rank can be adequately supplied with personnel, and if not, what changes need to be made to the structure. If the ranks can be adequately supplied, it is important to identify the time personnel are spending in a given rank before promotion. We aim to produce simple, practical measures that workforce planners can employ to make these determinations about their workforce structures.

\section{WORKFORCE MODEL \& LINEAR PROGRAMMING FORMULATION}

Because this work aims to analyse the higher-level dynamics of the Defence workforce, our simplified model encapsulates the fundamental structure and behaviour of the workforce but excludes important considerations that are present in reality. The inputs to the model are described in Table 1 and the decision variables in Table 2

In this model, the workforce is divided into independent trades, each modelled as a progression of ranks, $r \in \mathcal{R}$, through which personnel move unidirectionally. Because trades are treated as independent, we omit references to trade here. Each rank has a requirement profile $q_{r, t} ;$ the number of positions or personnel required to meet the operational function of the Defence service, at each timestep $t \in \mathcal{T}$. Personnel are handled in aggregate, but are split into groups according to their rank and time in rank $\rho \in \mathcal{P}$. The number of personnel in a given rank at a particular time and time in rank is called the supply $s_{r, t, \rho}$. These supply values are nonnegative real numbers, as we model the wastage (personnel leaving the workforce) deterministically rather than stochastically, by reducing the supply by the proportion $w_{r} \in[0,1]$ for each rank at each timestep. In effect, expected numbers of personnel rather than actual numbers are compared with requirement. This significant 
Table 1. The parameters and sets in the model.

\begin{tabular}{ll}
$\mathcal{T}$ & The set of monthly timesteps indexed by $t \in\{0, \ldots, T\}$. \\
$\mathcal{R}$ & The set of ranks indexed by $r \in\{1, \ldots, R\}$. \\
$\mathcal{P}$ & The set of potential times in rank indexed by $\rho \in\{0, \ldots, P\}$. \\
$\rho_{r}^{\text {min }}$ & Minimum time in rank $r \in \mathcal{R} \backslash\{R\}$, in months required to promote from rank $r$ to $r+1$. \\
$q_{r, t}$ & Number of personnel required in rank $r \in \mathcal{R}$ at time $t \in \mathcal{T}$. \\
$w_{r}$ & Proportion of personnel $\left(w_{r} \in[0,1]\right)$ in rank $r \in \mathcal{R}$ leaving the workforce each timestep. \\
$c_{r}^{\text {under }}$ & The cost (in arbitrary units) of undersupplying rank $r \in \mathcal{R}$ per person. \\
$c_{r}^{\text {over }}$ & The cost (in arbitrary units) of oversupplying rank $r \in \mathcal{R}$ per person. \\
\hline
\end{tabular}

Table 2. The decision variables in the model.

$\begin{array}{ll}s_{r, t, \rho} & \text { Non-negative, real number of personnel }\left(s_{r, t, \rho} \in \mathbb{R}_{\geq 0}\right) \text { in rank } r \in \mathcal{R} \text { at time } t \in \mathcal{T} \text { with time } \\ & \text { in rank } \rho \in \mathcal{P} . \\ p_{r, t, \rho} & \text { Non-negative, real number of personnel }\left(p_{r, t, \rho} \in \mathbb{R}_{\geq 0}\right) \text { that are promoted out of rank } \\ & r \in \mathcal{R} \backslash\{R\} \text { at time } t \in \mathcal{T} \text { with time in rank } \rho \in \mathcal{P} . \\ i_{t} \quad & \text { Non-negative, real number of personnel }\left(i_{t} \in \mathbb{R}_{\geq 0}\right) \text { recruited into the lowest rank } r=1 \text { at } \\ & \text { time } t \in \mathcal{T} . \\ s_{r, t}^{\text {under }} & \text { The non-negative amount of undersupply }\left(s_{r, t}^{\text {under }} \in \mathbb{R}_{\geq 0}\right) \text { in rank } r \in \mathcal{R} \text { at time } t \in \mathcal{T} . \\ s_{r, t}^{\text {over }} & \text { The non-negative amount of oversupply }\left(s_{r, t}^{\text {over }} \in \mathbb{R}_{\geq 0}\right) \text { in rank } r \in \mathcal{R} \text { at time } t \in \mathcal{T} .\end{array}$

simplifying assumption is reasonable in the context of the development of simple formulae and principles for potential use by planners in examining workforce structures. A more complex stochastic wastage model would complicate this problem while not adding much to the results.

The initial supply at $t=0$ is known. Each rank has a defined minimum time in rank (MTR) $\rho_{r}^{\min }$ : the absolute minimum amount of time that personnel must spend in that rank before being eligible for promotion to the next rank. While other factors can affect promotion decisions in reality, it is assumed here that minimum time in rank is the only consideration. Later work will consider models in which this assumption is relaxed. Our primary controls, then, are the promotions $p_{r, t, \rho}$ : the number of eligible personnel promoted from rank $r$ to rank $r+1$ at time $t$ with time in rank $\rho$. We assume that personnel can only enter the workforce via the lowest rank through inflow $i_{t}$. Both promotions $p_{r, t, \rho}$ and inflows $i_{t}$ are non-negative real numbers in keeping with supply $s_{r, t, \rho}$ being real numbers. Whilst these are the decision variables, this work focuses less on their determination and more on their relationship with other parameters.

In order to examine the relationship between requirement and supply for different model parameters, the recruitment and promotions need to be obtained. These are determined by formulating the problem as a pure linear program involving a weighted cost function based on the magnitude of undersupply (fewer personnel than requirement) and oversupply (more personnel than requirement). The formulation is presented below:

$$
\text { minimize } \quad \sum_{r, t} c_{r}^{\text {under }} s_{r, t}^{\text {under }}+c_{r}^{\text {over }} s_{r, t}^{\text {over }}
$$

subject to,

$$
\begin{aligned}
& s_{r, t}^{\text {under }} \geq q_{r, t}-\sum_{\rho} s_{r, t, \rho} \\
& s_{r, t}^{\text {under }} \geq 0 \\
& s_{r, t}^{\text {over }} \geq \sum_{\rho} s_{r, t, \rho}-q_{r, t} \\
& s_{r, t}^{\text {over }} \geq 0 \\
& s_{r, t, \rho}=\left(1-w_{r}\right) s_{r, t-1, \rho-1}-p_{r, t, \rho} \\
& s_{r, t, 0}=\sum_{\rho} p_{r-1, t, \rho} \\
& s_{1, t, 0}=i_{t} \\
& p_{r, t, \rho}=0
\end{aligned}
$$

$$
\begin{array}{r}
\forall r \in \mathcal{R}, t \in \mathcal{T} \\
\forall r \in \mathcal{R}, t \in \mathcal{T} \\
\forall r \in \mathcal{R}, t \in \mathcal{T} \\
\forall r \in \mathcal{R}, t \in \mathcal{T} \\
\forall r \in \mathcal{R}, t \in \mathcal{T} \backslash\{0\}, \rho \in \mathcal{P} \backslash\{0\} \\
\forall r \in \mathcal{R} \backslash\{1\}, t \in \mathcal{T} \backslash\{0\} \\
\forall t \in \mathcal{T} \\
\forall r \in \mathcal{R}, t \in \mathcal{T}, \rho \in\left\{0, \ldots, \rho_{r}^{\min }-1\right\}
\end{array}
$$

The cost function in (1) sums the weighted cost of undersupply and oversupply over all ranks and timesteps. Constraints (2)-(5) relate the gap between requirement and supply to the non-negative undersupply and nonnegative oversupply to maintain linearity of the cost function. Alternative cost functions could be implemented, but the simple cost function was sufficient for our purposes of moderating undersupply and oversupply. 
The flow equation (6) balances incoming and outgoing personnel at all times and ranks. It states that the supply in the next timestep is equal to the supply in the previous timestep minus wastage and promotions out of the rank. For zero time in rank - which occurs when personnel are promoted - the supply is equal to the promotions entering from the rank below as described in (7). For the lowest level, this is equal to the inflow at that time, as given in (8). Constraint (9) ensures that personnel with less time in rank than the MTR cannot be promoted. For the sake of clarity, other constraints in the formulation, such as those relevant to the initial state or the limits of time in rank, are not described but are straightforward to apply.

\section{OPTIMISATION RESULTS}

To demonstrate how the parameters affect supply, three simple test problems with different parameter variations representative of real four-rank workgroups in the RAN are discussed. All three problems are modelled over a 20-year period. The first problem has an initial requirement of 240, 150, 100, and 80 personnel for each rank that remains steady over five years, doubles over ten years and then remains steady for a further five years. The initial supply of personnel is set equal to this initial requirement. The MTR for all ranks is set to 48 months, and the wastage to $1 \%$ per month for all ranks. The second problem is a variation on the first with only a 50\% increase in requirement and 24 months MTR. The last problem is another variation on the first, but with no workforce growth and $1.7 \%$ monthly wastage.

Each test problem has two different variations of cost weightings. In both cases, undersupply is costed at 100, $200,300,400$ per person in each of the four ranks (from lowest to highest). The first variation costs oversupply at 1 per person independent of rank, while the second costs oversupply at 400, 800, 1200, 1600 per person in the four ranks (from lowest to highest). The first variation is chosen to ensure that under optimisation the requirement in all ranks is met, even if that results in an excess of personnel. The second variation enables us to observe the impact of not allowing such excesses.

The LP optimiser described in Section 2 is implemented in the Python programming language with the Google OR-Tools GLOP solver. The optimiser was run on each of the test problems using a standard desktop computer with an AMD Ryzen 7 2700X 8-core CPU. The simplicity of the model and the use of real rather than integer variables meant that the test problems could be run to optimality with run times between $47 \mathrm{~s}$ and $105 \mathrm{~s}$. The supply determined by the optimiser for the three problems and two cost variations is shown in Figure 1.
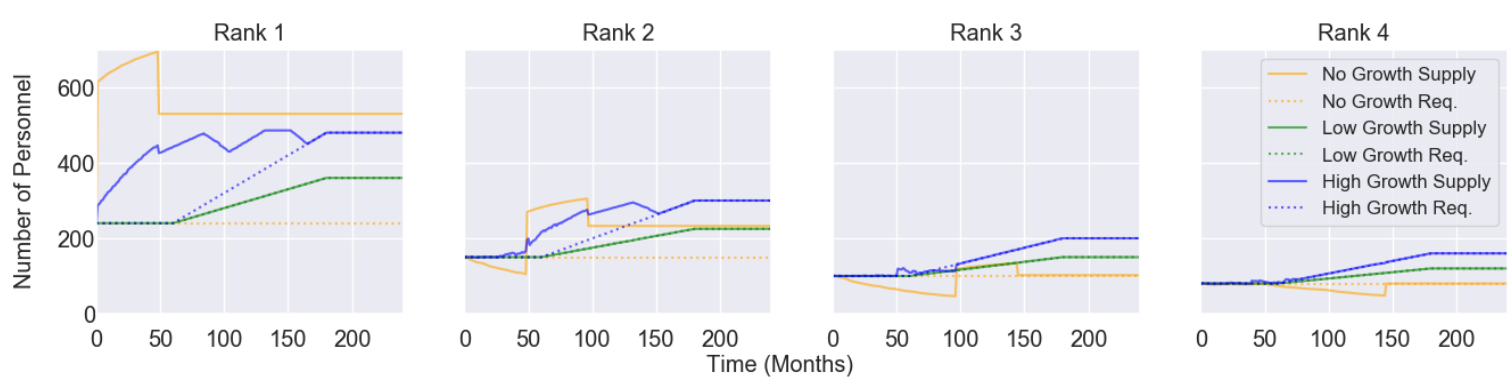

(a) Low oversupply costs of 1 per person for all ranks.
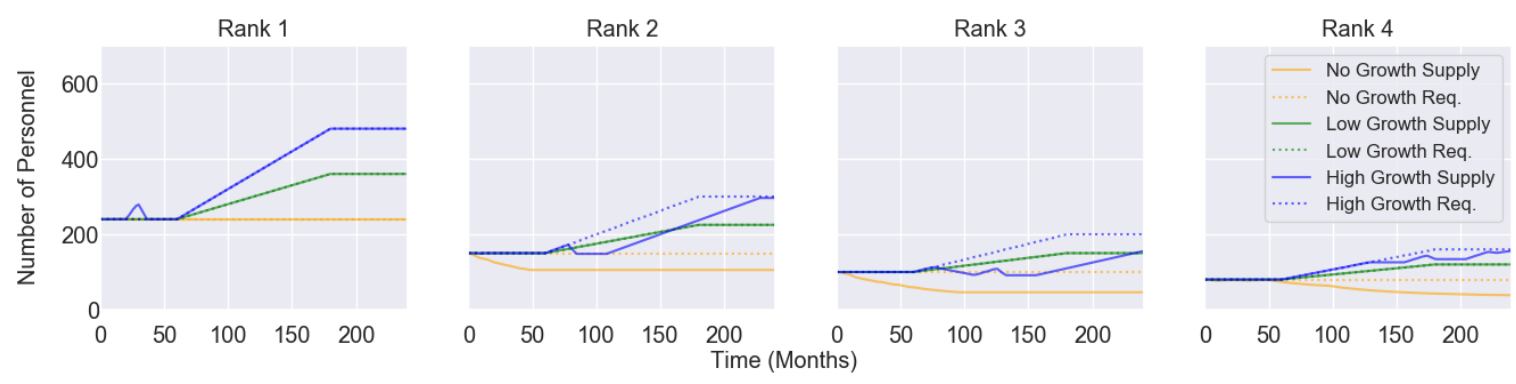

(b) High oversupply costs of 400, 800, 1200, 1600 per person for each of the ranks (from lowest to highest).

Figure 1. Optimised supply (solid) and requirement (dotted) with a) low oversupply costs and b) high oversupply costs for the three test problems: 100\% workforce growth (blue), 50\% workforce growth and 24 months MTR (green), and no growth but $1.7 \%$ monthly wastage (orange). 
From Figure 1(a) we observe that for some parameter settings, the optimiser has determined that it is necessary to oversupply some ranks to avoid undersupplies which have a much higher cost. This is confirmed by Figure 1(b), where higher oversupply costs reduce the oversupply in the optimal solution, and as a result, shortfalls of personnel occur. These results highlight a common problem in defence workforce planning, where simply training enough personnel to meet the requirement in the first rank leads to shortfalls in higher ranks in the future. Particular ranges of workforce parameters will force oversupply in some ranks in order to prevent large future shortfalls. It should be noted that workforce planners may consider such oversupplies too costly and may be willing to accept some higher rank undersupply to avoid large excesses. In such cases, it is up to the workforce planner to decide how to weight the cost function to represent their tolerance for oversupply and undersupply in particular ranks.

\section{STRUCTURE ANALYSIS}

It is apparent from the optimisation results that oversupplies can occur when the supply of a rank needs to be sufficiently high to sustain a certain required number of promotions out of the rank and prevent shortfalls in the higher ranks at later times. This leads us to the main focus for this paper: exploring how the requirement and other workforce parameters affect the need for this oversupply, and deriving simple measures to determine when such oversupplies are necessary and quantify their magnitude. Whilst the optimiser in Section 2 can be used to determine recruitment and promotion policies, it is limited in precisely identifying how the workforce parameters interact to cause this oversupply behaviour and the extent of these effects. Such knowledge provides utility to workforce planners in analysing the sustainability of their workforce structure.

We can assume that if an oversupply is needed in a rank, then personnel must be promoted from it as soon as they reach the minimum time in rank. If personnel were not promoted as soon as eligible, then they would be filling the higher ranks with less supply (due to wastage occurring over a longer time) and at later times (due to delayed promotion). In this situation, then, we can assume that personnel will be promoted as soon as they are eligible.

The necessary number of promotions for each rank and timestep can be calculated by determining the number of promotions needed to sustain the higher ranks. To do this, we approach the problem in reverse, by calculating the number required to sustain a rank $r$ from $t-1$ to $t$ so that the supply equals the requirement, and then backpropagating this required number of personnel through to each of the ranks below, taking into account wastage and assuming promotion only at the MTR. This is presented in (10) and (11), where the supply can be calculated from equations (6) and (7) and a sufficiently large $P$ is assumed so that the supply at $t-1$ is equal to the requirement at that time. Note that promotions from $r=0$ are permissible in (11), but these are equivalent to inflows where $p_{0, t, \rho_{0}^{\min }}=i_{t}$.

$$
\begin{aligned}
& 0=\sum_{\rho=0}^{P} s_{r, t, \rho}-q_{r, t}=s_{r, t, 0}+\sum_{\rho=1}^{P} s_{r, t, \rho}-q_{r, t} \\
&=p_{r-1, t, \rho_{r-1}^{\min }}+\sum_{\rho=1}^{P}\left[\left(1-w_{r}\right) s_{r, t-1, \rho-1}-p_{r, t, \rho}\right]-q_{r, t} \\
& p_{r-1, t, \rho_{r-1}^{\min }}=q_{r, t}-\left(1-w_{r}\right) q_{r, t-1}+p_{r, t, \rho_{r}^{\min }} \\
& p_{r-2, t-\rho_{r-1}^{\min }, \rho_{r-2}^{\min }}= \frac{q_{r, t}-\left(1-w_{r}\right) q_{r, t-1}+p_{r, t, \rho_{r}^{\min }}}{\left(1-w_{r-1}\right)^{\rho_{r-1}^{\min }}} \\
& p_{r-n, t-\sum_{i=2}^{n} \rho_{r-i+1}^{\min }, \rho_{r-n}^{\min }}=\frac{q_{r, t}-\left(1-w_{r}\right) q_{r, t-1}+p_{r, t, \rho_{r}^{\min }}}{\prod_{i=2}^{n}\left(1-w_{r-i+1}\right)^{\rho_{r-i+1}^{\min }}} \quad(2 \leq n \leq r)
\end{aligned}
$$

Promotions from each rank and time needs to be sufficient to supply all of the ranks above it. We let $p_{r, t, r+n}^{\prime}$ be the total promotions required from rank $r$ and time $t$ to sustain the rank $r+n$ where $1 \leq n \leq R-r$. Equating this to the formulas in (10) and (11), we obtain

$$
p_{r-1, t, r}^{\prime}:=p_{r-1, t, \rho_{r-1}^{\min }} \quad \text { and } \quad p_{r-n, t-\sum_{i=2}^{n} \rho_{r-i+1}^{\min }, r}^{\prime}:=p_{r-n, t-\sum_{i=2}^{n} \rho_{r-i+1}^{\min }, \rho_{r-n}^{\min }} \quad(2 \leq n \leq r)
$$

The required number of promotions $p_{r, t, \rho_{r}^{\min }}^{*}$ can then be calculated as,

$$
p_{r, t, \rho_{r}^{\min }}^{*}=\max \left(p_{r, t, r+1}^{\prime}, p_{r, t, r+2}^{\prime}, \ldots, p_{r, t, R}^{\prime}\right)
$$

We can also calculate the number of promotable personnel that can be produced by each rank. We make the simplifying assumption that personnel are only promoted after a fixed time in rank and only promotions from 
a single, unique time are relevant to this calculation. This implies that the distribution of time in rank is fixed. Our previous assumptions (personnel are promoted as soon as they reach the MTR and supply should equal the requirement) force a truncated geometric distribution (due to wastage) for the supply of personnel in the rank separated by time in rank. The upper bound of the support of this distribution is the MTR. Its supply at that value is the maximum number of promotable personnel $s_{r, t, \rho_{r}^{\min }}^{*}$ that can be produced by the rank without exceeding the requirement. This supply is calculated as follows.

$$
\begin{aligned}
& s_{r, t, \rho_{r}^{\min }}+s_{r, t-1, \rho_{r}^{\min }-1}+s_{r, t-2, \rho_{r}^{\min }-2}+\cdots+s_{r, t-\rho_{r}^{\min }, 0}=\sum_{i=0}^{\rho_{r}^{\min }} s_{r, t-i, \rho_{r}^{\min }-i} \\
& s_{r, t, \rho_{r}^{\min }}+\frac{s_{r, t, \rho_{r}^{\min }}}{1-w_{r}}+\frac{s_{r, t, \rho_{r}^{\min }}}{\left(1-w_{r}\right)^{2}}+\cdots+\frac{s_{r, t, \rho_{r}^{\min }}}{\left(1-w_{r}\right)_{r}^{\min }}=q_{r, t} \\
& s_{r, t, \rho_{r}^{\min }}^{*}:=s_{r, t, \rho_{r}^{\min }}=\frac{q_{r, t}}{\sum_{i=0}^{\rho_{r}^{\min }} \frac{1}{\left(1-w_{r}\right)^{i}}}=\frac{q_{r, t} w_{r}\left(1-w_{r}\right)^{\rho_{r}^{\min }}}{1-\left(1-w_{r}\right)_{r}^{\rho_{r}^{\min }}+1}
\end{aligned}
$$

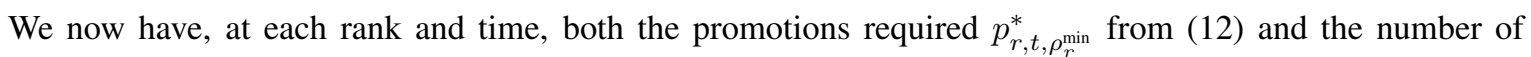
promotable personnel available $s_{r, t, \rho_{r}^{\min }}^{*}$ from (13). We refer to these as the Required Production Number (RPN) and the Suppliable Production Number (SPN), respectively. If the RPN is larger than the SPN, then for the given requirement, not enough promotions can be met, and a shortfall will, of necessity, appear in some of the upper ranks later. The requirement at the rank and time of interest is not enough to sustain the requirement of some of the higher ranks at a point in the future. The necessary requirement at $r$ to meet the RPN can be

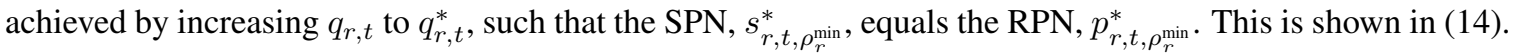

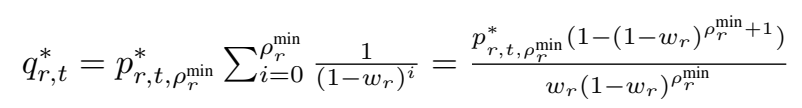

Conversely, if the SPN is larger than the RPN, then some promotable personnel will not need to be promoted and will spend longer in the rank. Along with our previous assumptions, if we assume that personnel must promote at a specific time in rank then we can calculate the time personnel will spend in rank before promotion by increasing the length of the support of the truncated geometric distribution until the last supply value equals the RPN, $p_{r, t, \rho_{r}^{\min }}^{*}$ For this new increased time in rank, $\rho_{r, t}^{*}$, we have

$$
\frac{q_{r, t}}{p_{r, t, \rho_{r}^{\min }}^{*}}=\frac{\sum_{i=0}^{\rho_{r, t}^{*}}\left(1-w_{r}\right)^{i}}{\left(1-w_{r}\right)^{\rho_{r, t}^{*}}}=\frac{1-\left(1-w_{r}\right)^{\rho_{r, t}^{*}+1}}{w_{r}\left(1-w_{r}\right)^{\rho_{r, t}^{*}}},
$$

so that

$$
\rho_{r, t}^{*}=\frac{-\ln \left(w_{r}\left(\frac{q_{r, t}}{p_{r, t, \rho_{r}^{\min }}^{*}}-1\right)+1\right)}{\ln \left(1-w_{r}\right)}
$$

At the start of this analysis, we assumed that personnel only promote at MTR and that supply will equal the requirement at all ranks. These assumptions can be removed by progressively calculating the SPN and RPN from the highest rank and earliest time to the lowest rank and latest time (progressing through times first and then ranks) and substituting the calculated $q_{r, t}^{*}$ for $q_{r, t}$ when the SPN is smaller than the RPN, and substituting the calculated $\rho_{r, t}^{*}$ for $\rho_{r}^{\min }$ when the SPN exceeds the RPN. By following this order of calculations, we can ensure that the RPN and SPN are calculated accurately (no assumption about the supply equalling the requirement or promotions at the minimum time in rank is made) with a single solution.

\section{DISCUSSION \& CONCLUSION}

Workforce planners can use equations (12)-(15) to calculate the RPN and SPN, as well as time to promotion and the required level of supply/requirement for each rank. These measures can provide useful insight into the structure of defence workforces. They can be used to determine whether a given requirement profile is sound and sustainable (i.e., it can exist without oversupplies or undersupplies over the long term). If the RPN exceeds the SPN, then either the number of personnel in some ranks must exceed the number of positions (or the requirement increased), or shortfalls will need to be accepted at particular times. Both situations represent a real cost to defence, with the former requiring supporting and paying for personnel that may not have a direct 
function, and the latter leading to decreased defence capability. If the SPN greatly exceeds the RPN, then the time to promotion may be significant enough to decrease morale, potentially leading to increased wastage.

The derived measures directly relate how rank requirement profiles, wastage and minimum time in rank interact to cause potential workforce issues. Whilst the formulae may appear complicated, they can be implemented in spreadsheet tools which are accessible to most planners. It is also possible to incorporate additional workforce dynamics into the equations, such as mid-career inflows and length of service dependent wastage. While the details of doing this are not presented here, their addition does not significantly change the formulae.

Importantly, these measures assume that supply in all ranks is not exceeded by requirement. Workforce planners may decide that it is not worth increasing the supply/requirement to avoid shortfalls in some ranks. In those cases, the cost of oversupplying particular ranks needs to be weighed against the cost of undersupplying others. This decision needs to be made by the workforce planner, but the LP optimisation formulation described in Section 2 can be used for that. The optimiser may also be easier to apply, especially in cases such as complex requirement profiles and time in rank distributions. The optimisation results can indirectly provide the same measures as the equations, but the equations may allow for quicker what-if analyses and better understanding of a particular supply profile. Regardless of how the values are obtained, they represent important measures that workforce planners can use to ensure their workforces have the right structure.

Two different types of requirement emerge from the analysis. The first is the requirement for actual positions in the defence service in order for that service to operate. We will term this as the functional requirement and it is an input to the model. But the second is the requirement profile needed in order to sustain the functional requirement for a given costing of undersupply and oversupply. We will term this as the structural requirement and it may be above or below the functional requirement at different times. The structural requirement can be obtained by either running an optimiser like the one described in Section 2 or by directly calculating the necessary requirement for sustainability as described in Section 4.

These factors are especially important to the RAN, where large growth is occurring as a result of major acquisitions. This intense growth will likely create the situations analysed in this work, where an initial large excess of personnel in lower ranks is necessary in order to avoid a large shortfall of personnel later on in higher ranks. Recruitment and training establishments should not target the requirement of lower ranks in the short term, but should aim to meet the structural requirement necessary for all of the ranks in the long term. Failure to adequately supply the early ranks could mean that Defence platforms may be rendered temporarily redundant. It is crucial that workforce planners analyse their workforce structures to ensure sustainability into the future.

\section{REFERENCES}

Bastian, N.D., Lunday, B.J., Fisher, C.B., Hall, A.O., 2020. Models and methods for workforce planning under uncertainty: Optimizing U.S. army cyber branch readiness and manning. Omega 92, 1-13.

Filinkov, A., Richmond, M., Nicholson, R., Alshansky, M., Stewien, J., 2011. Modelling personnel sustainability: a tool for military force structure analysis. Journal of the Operational Research Society 62, 1485-1497.

Gass, S.I., 1991. Military manpower planning models. Computers \& Operations Research 18, 65-73.

Hall, A.O., 2009. Simulating and optimizing: Military manpower modeling and mountain range options. Ph.D. thesis. University of Maryland.

Horn, M.E.T., Gomez-Iglesias, A., 2016. Strategic workforce planning for the Australian Defence Force. Journal of the Operational Research Society 67, 664-675.

Moorhead, P., Wind, A., Halbrohr, M., 2008. A discrete event simulation model for examining future sustainability of Canadian Forces operations, in: Proceedings of the 40th Conference on Winter Simulation, Winter Simulation Conference. pp. 1164-1172.

Ozakawa, S., Straver, M., Arseneau, L., 2018. Occupation Promotion Recruitment and Attrition Model (OPRAM). Technical Report. DRDC. Ottawa, Canada.

Scales, C., Okazawa, S., Ormrod, M., 2011. The managed readiness simulator: A force readiness model, in: Proceedings of the 2011 Winter Simulation Conference (WSC), pp. 2514-2524.

Turan, H., Elsawah, S., Ryan, M., 2019. Simulation-based analysis of military workforce planning strategies, in: MSIE 2019, pp. 68-75.

Weigel, H.S., Wilcox, S.P., 1993. The army's personnel decision support system. Decision Support Systems 9, 281-306.

Wesolkowski, S., Eisler, C., 2015. Capability-Based Models for Force Structure Computation and Evaluation. Technical Report. DRDC. Ottawa, Canada. 